\title{
ON INCIDENCE ALGEBRAS AND DIRECTED GRAPHS
}

\author{
ANCYKUTTY JOSEPH
}

Received 20 June 2001

\begin{abstract}
The incidence algebra $I(X, \mathbb{R})$ of a locally finite poset $(X, \leq)$ has been defined and studied by Spiegel and O'Donnell (1997). A poset $(V, \leq)$ has a directed graph $\left(G_{v}, \leq\right)$ representing it. Conversely, any directed graph $G$ without any cycle, multiple edges, and loops is represented by a partially ordered set $V_{G}$. So in this paper, we define an incidence algebra $I(G, \mathbb{Z})$ for $(G, \leq)$ over $\mathbb{Z}$, the ring of integers, by $I(G, \mathbb{Z})=\left\{f_{i}, f_{i}^{*}: V \times V \rightarrow \mathbb{Z}\right\}$ where $f_{i}(u, v)$ denotes the number of directed paths of length $i$ from $u$ to $v$ and $f_{i}^{*}(u, v)=-f_{i}(u, v)$. When $G$ is finite of order $n, I(G, \mathbb{Z})$ is isomorphic to a subring of $M_{n}(\mathbb{Z})$. Principal ideals $I_{v}$ of $(V, \leq)$ induce the subdigraphs $\left\langle I_{v}\right\rangle$ which are the principal ideals $\Phi_{v}$ of $\left(G_{v}, \leq\right)$. They generate the ideals $I\left(\Phi_{v}, \mathbb{Z}\right)$ of $I(G, \mathbb{Z})$. These results are extended to the incidence algebra of the digraph representing a locally finite weak poset both bounded and unbounded.
\end{abstract}

2000 Mathematics Subject Classification: 05C20, 06A11.

1. Introduction. The incidence algebra $I(X, \mathbb{R})$ of a locally finite poset $X$ over a commutative ring $\mathbb{R}$ with identity is discussed in much detail by Spiegel and O'Donnell [5]. Every poset $(V, \leq)$ has a directed graph $\left(G_{v}, \leq\right)$, or $(G, \leq)$ for short, representing it. The directed graph $(G, \leq)$ is free of cycles and multiple arcs. It is natural for $(G, \leq)$ to have an incidence algebra whose properties depend on those of the directed graph.

In this paper, we define and study an incidence algebra $I(G, \mathbb{Z})$ for $(G, \leq)$ over the ring $\mathbb{Z}$ of all integers. Section 2 contains the basic results and definitions used in this paper. Section 3 deals with $I(G, \mathbb{Z})$, the incidence algebra of $(G, \leq)$ representing a finite poset $(V, \leq)$ of cardinality $n$. Ideals of $I(G, \mathbb{Z})$ are given through the principal ideals of $(G, \leq)$ which are the subdigraphs induced by the principal ideals of $(V, \leq)$. An extension of the results given in Section 3 to the digraph representing a locally finite weak poset $(V, \leq)$ bounded or unbounded is the content of Section 4 .

2. Definitions and basic results. We consider the directed graph $\left(G_{v}, \leq\right)$ representing a locally finite partially ordered set $(V, \leq)$. The terminology introduced here is used throughout the paper.

DeFinITION 2.1. For any $u \leq v$ in $(V, \leq),[u, v]=\{w: u \leq w \leq v\}$ is an interval of $(V, \leq)$. The length of $[u, v]$ denoted by $l[u, v]$ is the length of the longest chain in $[u, v]$. A poset $(V, \leq)$ is locally finite if $l[u, v]$ is finite for each $[u, v]$ in $(V, \leq)$; and $(V, \leq)$ is bounded if there is a $k>0$ such that $l[u, v] \leq k$ for all $[u, v]$ in $(V, \leq)$. Otherwise $(V, \leq)$ is unbounded. 
DEFINITION 2.2. The directed graph $\left(G_{v}, \leq\right)$ associated with a locally finite poset $(V, \leq)$ is defined as $\left(G_{v}, \leq\right)=(V, E)$ where $V=(V, \leq)$ and $E=\{\operatorname{arcs} \overrightarrow{u v} ; u<v\}$; $\left(G_{v}, \leq\right)$ has no cycles nor multiple arcs.

LEMMA 2.3 (see [5]). A finite poset $(V, \leq)$ can be labelled $V=\left\{v_{1}, v_{2}, \ldots, v_{n}\right\}$ so that $v_{i} \leq v_{j}$ implies that $i \leq j$.

Proposition 2.4 (see [4]). The vertices of a finite directed graph $G=(V, E)$ can be labelled $v_{1}, v_{2}, \ldots, v_{n}$ such that $\vec{v}_{i} \vec{v}_{j} \in E$ implies that $i<j$, if and only if $G$ has no cycles.

Note 2.5. Lemma 2.3 and Proposition 2.4 have motivated the orientation of arcs in $\left(G_{v}, \leq\right)$.

DEFINITION 2.6 (see [5]). Let $(X, \leq)$ be a locally finite poset and $\mathbb{R}$ a commutative ring with identity. The incidence algebra $I(X, \mathbb{R})$ of $X$ over $\mathbb{R}$ is defined by

$$
I(X, \mathbb{R})=\{f: X \times X \rightarrow \mathbb{R}: f(x, y)=0 \text { if } x \nless y\},
$$

with operations defined by

(i) $(f+g)(x, y)=f(x, y)+g(x, y)$;

(ii) $(f \cdot g)(x, y)=\sum_{x \leq u \leq y} f(x, u) g(u, y)$;

(iii) $(r f)(x, y)=r f(x, y)$, for all $r \in \mathbb{R} ; f, g \in I(X, \mathbb{R})$.

Definition 2.7. For any $v \in(V, \leq)$, let $I_{v}=\{u \in V: u \leq v\}$; $I_{v}$ is called the principal ideal generated by $v$.

DEFINITION 2.8. An ideal $\mathscr{I}$ of $\left(G_{v}, \leq\right)$ is an induced subdigraph of $G_{v}$ such that all directed paths with their terminal vertex in $\mathscr{I}$ are in $\mathscr{I}$.

If $I_{v}$ is a principal ideal of $(V, \leq),\left\langle I_{v}\right\rangle$, the subdigraph induced by $I_{v}$ is the principal ideal of $G$ generated by $v$ in $\left(G_{v}, \leq\right)$. Denote $\left\langle I_{v}\right\rangle$ by $\Phi_{v}$.

NotATION 2.9. For the remaining part of the paper, the digraphs $(G, \leq)$ and $\left(G_{\infty}, \leq\right)$ represent the finite poset $(V, \leq)$ and the locally finite poset $\left(V_{\infty}, \leq\right)$, respectively.

3. An incidence algebra for $(G, \leq)$. In this section, we define an incidence algebra $I(G, \mathbb{Z})$ for the digraph $(G, \leq)$ representing the finite poset $(V, \leq)$. Subalgebras and ideals of $I(G, \mathbb{Z})$ are defined through principal ideals of $(G, \leq)$. Assume that $V=\left\{v_{1}, v_{2}, \ldots, v_{n}\right\}$.

DeFinITION 3.1. For $u, v \in V$, let $p_{k}(u, v)$ denote the number of directed paths of length $k$ from $u$ to $v$ and $p_{k}(v, u)=-p_{k}(u, v)$.

For $i=0,1,2, \ldots, n-1$, define $f_{i}, f_{i}^{*}: V \times V \rightarrow \mathbb{Z}$ by

$$
f_{i}(u, v)=p_{i}(u, v), \quad f_{i}^{*}(u, v)=-p_{i}(u, v) .
$$

The incidence algebra $I(G, \mathbb{Z})$ of $(G, \leq)$ over the commutative ring $\mathbb{Z}$ with identity is defined by $I(G, \mathbb{Z})=\left\{f i, f i^{*}: V \times V \rightarrow \mathbb{Z}, i=0,1,2, \ldots, n-1\right\}$ with operations defined as follows:

(i) for $f \neq g,(f+g)(u, v)=f(u, v)+g(u, v)$; 
(ii) $(f \cdot g)(u, v)=\sum_{w} f(u, w) g(w, v)$;

(iii) $(z f)(u, v)=z f(u, v)$, for all $z \in \mathbb{Z} ; f, g \in I(G, \mathbb{Z})$.

REMARK 3.2. The function $f_{l}$ is the digraph analogues of $\chi \in I(X, \mathbb{R})$ [5]. The ma$\operatorname{trix}\left[f_{l},\left(v_{i}, v_{j}\right)\right]$ is the adjacency matrix of $(G, \leq)$ and $f_{l}^{k}\left(v_{i}, v_{j}\right)=f_{k}\left(v_{i}, v_{j}\right)$. For any interval $[u, v]$ with $l[u, v]=k, f_{l}^{k}(u, v)=f_{k}(u, v)=0$. For every $f \in I(G, \mathbb{Z})$, there is a constant $m \in \mathbb{Z}$ such that $f^{m}(u, v)=0$, for all $(u, v) \in V \times V$.

DEFINITION 3.3. With each ideal $\Phi_{v}=\left\langle I_{v}\right\rangle$ of $(G, \leq)$ we associate an incidence algebra,

$$
\begin{gathered}
I\left(\Phi_{v}, \mathbb{Z}\right)=\left\{f \in I(G, \mathbb{Z}): f: I_{v} \times I_{v} \longrightarrow \mathbb{Z}\right\}, \\
\forall f \in I\left(\Phi_{v}, \mathbb{Z}\right), f\left(v_{i}, v_{j}\right)=0 \quad \forall\left(v_{i}, v_{j}\right) \notin I_{v} \times I_{v} .
\end{gathered}
$$

REMARK 3.4. If $(H, \leq)$ is a subdigraph of $(G, \leq)$, then $I(H, \mathbb{Z})$ is a subalgebra of $I(G, \mathbb{Z})[5]$. In particular, $I\left(\Phi_{v}, \mathbb{Z}\right)$ is a subalgebra of $I(G, \mathbb{Z}) ; I\left(\Phi_{v}, \mathbb{Z}\right)$ is called the subalgebra generated by the vertex $v$.

REMARK 3.5 (see [5]). If $S$ is an ideal of $\mathbb{Z}, I(G, S)=\{f \in I(G, \mathbb{Z}) ; f(u, v) \in S\}$ is a subalgebra of $I(G, \mathbb{Z})$.

Proposition 3.6. For each principal ideal $\Phi_{v}$ of $(G, \leq), I\left(\Phi_{v}, \mathbb{Z}\right)$ is an ideal of the $\operatorname{ring} I(G, \mathbb{Z})$.

Proof. Let $\Phi_{v}$ be a proper principal ideal of $(G, \leq)$. Denote the elements of $I(G, \mathbb{Z})$ and $I\left(\Phi_{v}, \mathbb{Z}\right)$ by $f$ and $f^{\prime}$, respectively. For all $f^{\prime} \in I\left(\Phi_{v}, \mathbb{Z}\right)$, there is a unique $f \in I(G, \mathbb{Z})$ such that $f^{\prime}(u, w)=f(u, w)$ for all $(u, w) \in I_{v} \times I_{v}$; and $f^{\prime}(u, w)=0$ for all $(u, w) \notin I_{v} \times I_{v}$.

Hence for any $(u, w) \in V \times V, f, g \in I(G, \mathbb{Z}), g^{\prime} \in I\left(\Phi_{v}, \mathbb{Z}\right)$

$$
\left(f g^{\prime}\right)(u, w)= \begin{cases}(f, g)(u, w), & \text { if }(u, w) \in I_{v} \times I_{v} \\ 0, & \text { otherwise }\end{cases}
$$

with similar values for $\left(g^{\prime} f\right)(u, w)$ also.

Consequently, $f g^{\prime}$ and $g^{\prime} f \in I\left(\Phi_{v}, \mathbb{Z}\right)$, and $I\left(\Phi_{v}, \mathbb{Z}\right)$ is an ideal of $I(G, \mathbb{Z})$.

REMARK 3.7. The incidence algebra $I(G, \mathbb{Z})$ is isomorphic to a subring of the ring of upper triangular matrices over $\mathbb{Z}$ [5]. In general, every ideal of $M_{n}(\mathbb{Z})$ has the form $M_{n}(S)$ for some ideal $S$ of $\mathbb{Z}$ [3].

PROPOSITION 3.8. Every ideal of $I(G, \mathbb{Z})$ has the form $I\left(\Phi_{v}, \mathbb{Z}\right)$ for some principal ideal $\Phi_{v}$ of $(G, \leq)$.

Proof. Let $S$ be a proper ideal of the $\operatorname{ring} I(G, \mathbb{Z})$. Then $S=I(H, \mathbb{Z})$ for some subdigraph $(H, \leq)$ of $(G, \leq)$. For all $f \in I(G, \mathbb{Z})$ and $g^{\prime} \in S, f g^{\prime}=g^{\prime} f \in S$.

Consequently, there is an $h^{\prime} \in S$ such that $f g^{\prime}=h^{\prime}$ satisfying $\left(f g^{\prime}\right)(u, v)=$ $h^{\prime}(u, v)=p_{k}(u, v)$ for some $k$ and for all $u, v$ in $V(H)$. 
Then for all $(u, v) \in V(H) \times V(H)$, the number of paths of length $k$ in $H$ from $u$ to $v$ is the same as that in $G$.

Hence for any $v \in V(H), H$ contains all the directed paths terminating in $v$. Then $H=\Phi_{v}$ for some $v$.

4. The incidence algebra $I\left(G_{\infty}, \mathbb{Z}\right)$. An extension of Proposition 2.4 is obtained by the author for locally finite directed graphs [2]. This provides an isomorphism between $I\left(G_{\infty}, \mathbb{Z}\right)$ and a subring of the ring of upper triangular matrices over $\mathbb{Z}$. Also we have extended Propositions 3.6 and 3.8 to bounded as well as unbounded locally finite weak posets. It is assumed that the poset $(V, \leq)$ is countable.

DEFINITION 4.1. A locally finite poset $(V, \leq)$ is weak if only finitely many chains intersect at every element $v$ in $V$.

DEFINITION 4.2. A directed graph $G=(V, E)$ is locally finite if every vertex is of a finite degree.

Definition 4.3. A vertex $v$ of the directed graph $\left(G_{\infty}, \leq\right)$, such that $d(v) \neq 0$, is called a source or atom if there is no other vertex $u$ such that $u \leq v$.

Proposition 4.4 (see [2]). Let $G=(V, E)$ be a locally finite, acyclic digraph where $V$ is countable; $V$ can be labelled $\left\{v_{1}, v_{2}, v_{3}, \ldots\right\}$ such that $\vec{v}_{i} \vec{v}_{j} \in E$ implies that $i<j$ if and only if

(i) the set $S$ of its sources is nonempty and finite;

(ii) for each $v \in V$ and $s \in S$ such that $\overrightarrow{s v} \in E$, any maximal directed path terminating in $v$ is of finite length.

DEFinITION 4.5. Let $(V, \leq)$ be a locally finite weak poset and $\left(G_{\infty}, \leq\right)$ the digraph representing $(V, \leq)$. The incidence algebra $I\left(G_{\infty}, \mathbb{Z}\right)$ of $\left(G_{\infty}, \leq\right)$ over the ring $\mathbb{Z}$ of integers is given by

$$
I\left(G_{\infty}, \mathbb{Z}\right)=\left\{f_{i}, f_{i}^{*}: V \times V \longrightarrow \mathbb{Z}\right\}
$$

satisfying the operations given in Definition 3.1.

DefinItion 4.6. An infinite matrix $A=\left[a_{i j}\right]$ is row (column) finite if $a_{i j} \neq 0$ for finitely many $j(i)$.

Note 4.7. (i) Each $f \in I\left(G_{\infty}, \mathbb{Z}\right)$ is represented by a matrix $[f]$ where $[f]_{i, j}=$ $f\left(v_{i}, v_{j}\right)$ and $[f]$ is both row and column finite, $M_{\infty}(\mathbb{Z})$ denotes the ring of row and column finite matrices over $\mathbb{Z}$. The incidence algebra $I\left(G_{\infty}, \mathbb{Z}\right)$ is isomorphic to a subring of $M_{\infty}(\mathbb{Z})$.

(ii) A characterization of infinite directed graphs for which $[f]$ is nilpotent is obtained by the author in [1].

Proposition 4.8. Let $(V, \leq)$ be a weak, locally finite poset satisfying the following:

(i) the set $S$ of its atoms is nonempty and finite;

(ii) for each $v \in V, s \in S$ such that $s \leq v$, any chain with $v$ as upperbound is of finite length. Then $I\left(G_{\infty}, \mathbb{Z}\right)$ is isomorphic to a subring of the ring of upper triangular matrices over $\mathbb{Z}$. 
Proof. The digraph $\left(G_{\infty}, \leq\right)$ is locally finite. By Proposition $4.4, f\left(v_{r}, v_{s}\right) \geq 0$ for all $r \leq s$ and for all $f \in I\left(G_{\infty}, \mathbb{Z}\right)$. Hence every $f$ has a representation as an upper triangular matrix over $\mathbb{Z}$.

DEFinition 4.9. In a bounded weak, locally finite poset $(V, \leq)$ a principal ideal $I_{v}=\{u: u \leq v\}$. The corresponding principal ideal of $\left(G_{\infty}, \leq\right)$ is given by $\Phi_{v}=\left\langle I_{v}\right\rangle$.

Proposition 4.10. Let $(V, \leq)$ be any weak, locally finite bounded poset and $\Phi_{v} a$ principal ideal of $\left(G_{\infty}, \leq\right)$. Then

(1) $I\left(\Phi_{v}, \mathbb{Z}\right)$ is a subalgebra of $I\left(G_{\infty}, \mathbb{Z}\right)$;

(2) $I\left(\mathscr{I}_{v}, \mathbb{Z}\right)$ is an ideal of $I\left(G_{\infty}, \mathbb{Z}\right)$.

NoTE 4.11. This follows from Propositions 3.6 and 4.4.

Proposition 4.12 (see [5]). Let $(V, \leq)$ be an unbounded weak poset. Then $V$ contains a subpartially ordered set isomorphic to $\mathbb{Z}^{+}, \mathbb{Z}^{-}$, or UCn where $C n$ denotes a chain of length $n$.

REMARK 4.13. When $(V, \leq)$ is unbounded, the principal ideals of $(V, \leq)$ and $\left(G_{\infty}, \leq\right)$ are not well defined. Hence Proposition 4.4 is not true for unbounded posets, in general.

But there are unbounded, locally finite posets satisfying Proposition 4.4. As an example we have $(V, \leq)=\left(\mathbb{Z}^{+}\right.$, usual ordering $)$.

Let $(V, \leq)$ be an unbounded, locally finite, weak poset such that $I\left(G_{\infty}, \mathbb{Z}\right)$ is isomorphic to a subring of the ring of upper triangular matrices. Then

(i) principal ideals of $\left(G_{\infty}, \leq\right)$ are defined as $\mathscr{I}_{v}=\left\langle I_{v}\right\rangle$;

(ii) for each $\Phi_{v}, I\left(\Phi_{v}, \mathbb{Z}\right)$ is a subalgebra of $I\left(G_{\infty}, \mathbb{Z}\right)$;

(iii) for every $\mathscr{I}_{v}, I\left(\mathscr{I}_{v}, \mathbb{Z}\right)$ is an ideal of $I\left(G_{\infty}, \mathbb{Z}\right)$.

ACKNOWLedgment. I would like to express my sincere gratitude to Prof. T. Thrivikraman, Head of the Department of Mathematics, Cochin University of Science and Technology, Kerala, India for his many valuable suggestions and continuing support throughout the preparation of this paper. Research under IX plan FIP of the UGC.

\section{REFERENCES}

[1] J. Ancykutty, On Nilpotent operators of infinite digraphs, submitted to J. Graph Theory.

[2] _ Logical numbering of infinite acylic digraphs, Journal of Tripura Mathematical Society 3 (2001), 21-28.

[3] N. Jacobson, Basic Algebra, vol. 1, Hindustan Publication Corporation, India, 1980.

[4] K. R. Parthasaradhy, Basic Graph Theory, Tata McGraw Hill, 1994.

[5] E. Spiegel and C. J. O'Donnell, Incidence Algebras, Monographs and Textbooks in Pure and Applied Mathematics, vol. 206, Marcel Dekker, New York, 1997.

ANCYKUTTY JOSEPH: DePARTMENT OF MATHEMATICS, ST. DOMINIC'S COLLEGE, KANJIRAPALLY 686512, KERALA, INDIA

E-mail address: ancykutty@rediffmai1.com 


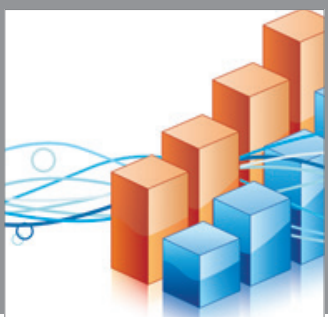

Advances in

Operations Research

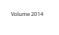

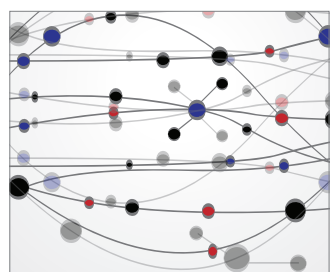

\section{The Scientific} World Journal
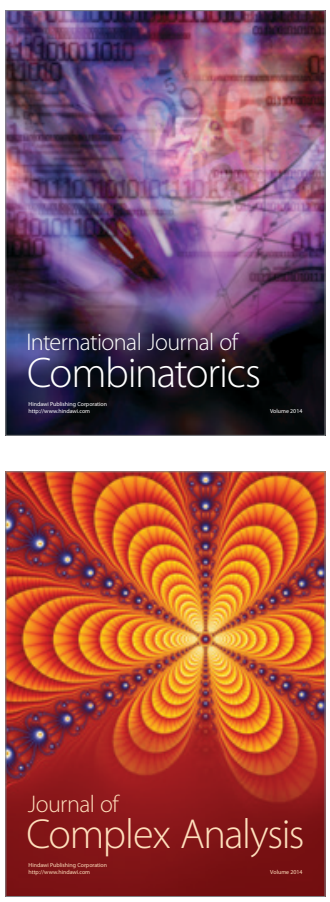

International Journal of

Mathematics and

Mathematical

Sciences
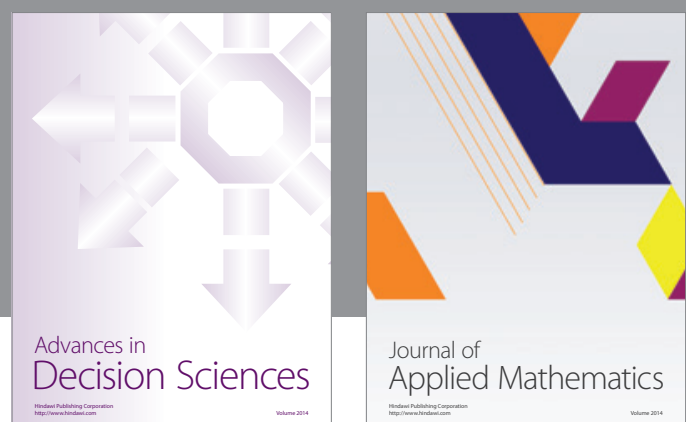

Journal of

Applied Mathematics
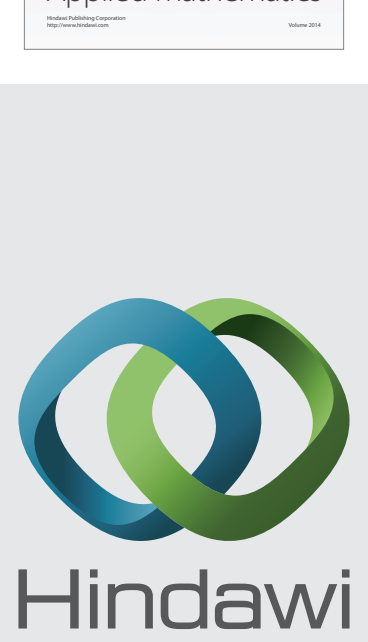

Submit your manuscripts at http://www.hindawi.com
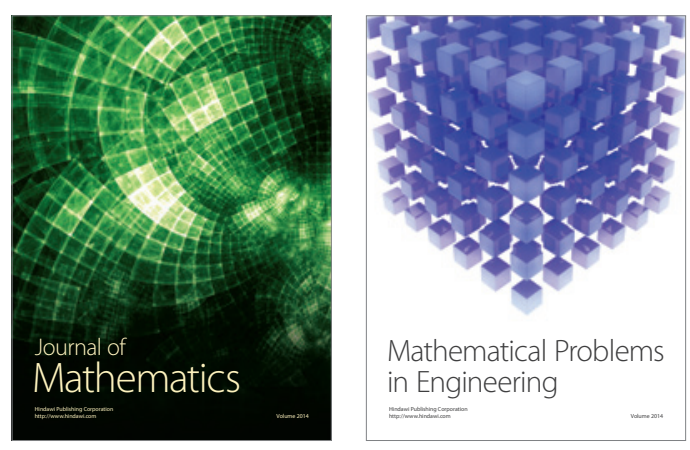

Mathematical Problems in Engineering
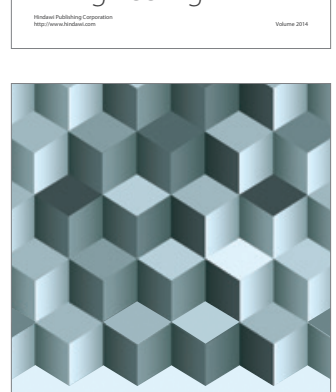

Journal of

Function Spaces
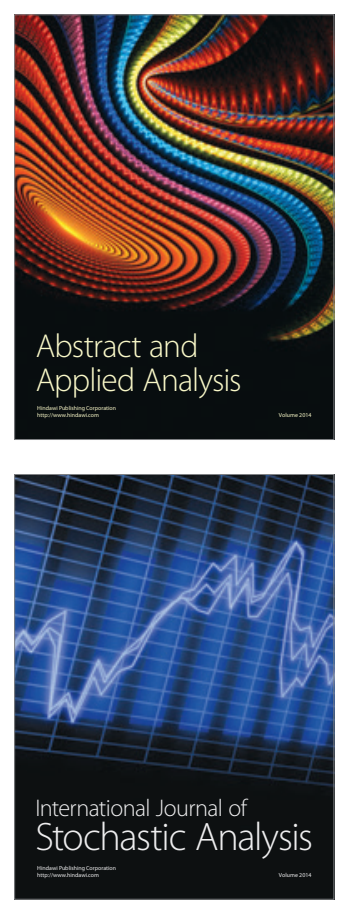

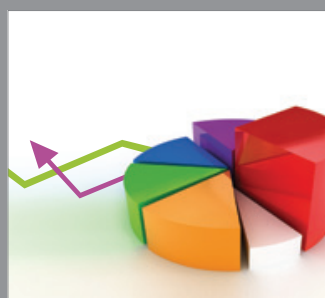

ournal of

Probability and Statistics

Promensencen
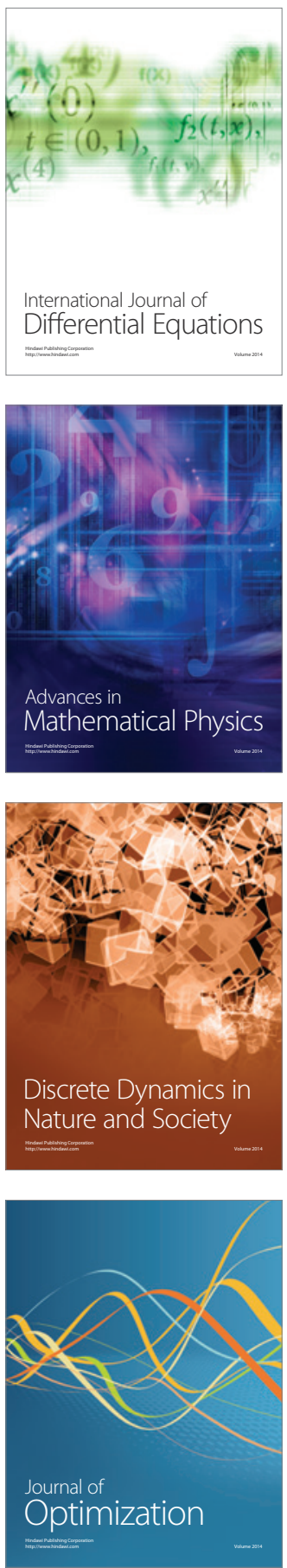